\title{
Early availability of laboratory results increases same day ward discharge rates
}

Michael P. Cornes, Graham Danks, Sanna Elgaddal, Mohammed Jawad, Jayne Tonks,

Elisabeth Ries, Clare Ford and Rousseau Gama

\section{Abstract}

Background: Delayed discharge reduces hospital efficiency and inconveniences patients. Most hospitals discharge in the afternoon, the most common admission time is mid-morning. Consequently, new patients wait for the beds of patients who are fit to be discharged. Earlier discharge may, therefore, improve patient flow. We investigated the impact of early phlebotomy with early availability of laboratory results on patient discharge rates and discharge time.

Methods: Discharge rates, discharge time and sample turnaround time were assessed before (1 October 2014 to 31 December 2014) and after (1 October 2015 to 31 December 2015) introduction of earlier phlebotomy with availability of laboratory results prior to the ward rounds on two surgical wards.

Results: Following the intervention, over 95\% of results were available before 8:30 am in 2015 as compared to less than $1 \%$ in 2014 . Specimen turnaround times were similar in both study periods. Even after adjustment for age, gender, admission type and length of admission, the same day discharge rate was higher in 2015 compared to 2014 (60\% vs. 52\%; $p<0.002$ ), but time of discharge was unchanged.

Conclusions: Early availability of blood results prior to ward rounds increased ward discharges but did not affect discharge time.

Keywords: biochemistry; discharge; hematology phlebotomy.

\section{Main messages}

— An earlier phlebotomy round results in the availability of results in time for ward rounds

- Earlier availability of laboratory results leads to increased same day discharge rates

— Earlier availability of laboratory results has no impact on discharge time of day

\section{Research questions}

— Does receiving samples earlier to the laboratory result in earlier availability of tests?

—Does earlier availability of laboratory results lead to earlier discharge time of patients?

—Does earlier availability of laboratory results lead to more same day patient discharges?

\section{Introduction}

Over recent years, the number of patient admissions to hospitals in the United Kingdom (UK) has increased whereas the number of available beds has declined, increasing the need for efficient but safe discharge of patients. Delayed safe discharge, or inappropriate hospital bed occupancy, is a 
common long-standing problem in the UK [1], which until recently has received scant attention because the emphasis has been on admission rates rather than discharge rates. Delayed discharge, however, carries a large cost to the NHS, reduces hospital efficiency and inconveniences patients. Audits have, for example, shown that hospitals with higher bed occupancy have higher rates of cancelled operations and are more likely to have a "bed crisis" [2]. In 2000, the National Audit Office reported that in the UK an estimated 600 patients per day were affected by delayed discharge at a cost of $£ 170$ million per year [2]. This is likely to have increased.

Another problematic feature of hospital admission and discharge patterns in the UK is that most discharges occur in the afternoon whereas the most common admission time is mid-morning [3]. Consequently, admitted patients wait for beds while those currently occupying them wait to see if they can be discharged home or to social care. Earlier discharge may, therefore, improve patient flow.

There are many factors that may influence discharge rates and discharge time. These include the following:

— Ability of the patient to care for themselves

- Availability of pharmacy services to review and prepare medication on discharge

- Availability of transport from the hospital to the patient's home

- Availability of family members to continue care at home

— Availability of social care services

— Completion of discharge paperwork by relevant healthcare staff

- Availability of laboratory test results to inform discharge decision making

Earlier availability of laboratory test results, a process controlled by the hospital, may facilitate hospital discharge and earlier planned discharge. Reports on the impact of pathology result availability and delayed discharge are conflicting. Some studies report a significant contribution to delayed discharge [4-6], whereas others report little or no effect $[7,8]$. In addition, there is a widely held perception amongst clinicians that late availability of pathology results delays discharge of patients. Phlebotomy earlier in the day, however, seems to have no impact on discharge time [9], but in this study there was no laboratory intervention to facilitate the turnaround of the earlier samples.

We, therefore, investigated the impact on patient discharge of early phlebotomy with early availability of laboratory results prior to the ward rounds.

\section{Materials and methods}

\section{Setting, patients and intervention}

This quality improvement project was undertaken on two surgical wards at New Cross Hospital, Royal Wolverhampton NHS Trust, UK. New Cross Hospital is a 700-bed teaching hospital serving a 
Multi-ethnic population of about 500,000 in one of the most deprived areas in the UK [10]. The phlebotomy service is part of the pathology directorate. Over $80 \%$ of blood science requests are electronic, and results are available on the hospital IT system as soon as they are analyzed.

In October 2015, an early 6:00 am phlebotomy ward round was introduced on the two 25-bed surgical wards supported by an early start $(6: 30 \mathrm{am})$ by staff involved in transporting samples to the laboratory (runners) and by an additional early laboratory staff member starting at 7:00 am to process the samples. Prior to intervention, the phlebotomy ward round began at 7:00 am, runners at 7:30 am and laboratory staff at 8:00 am.

Changes were assessed by comparing differences in patient data before (1 October 2014 to 31 December 2014) and after (1 October 2015 to 31 December 2015) the intervention.

Data collected from the laboratory information management system for each study period included date and time of receipt of blood samples in the laboratory, age, gender and date and time blood results were available to clinicians. Data collected from the hospital informatics team included patient discharge dates and times, admission type and admission time. Admission type was categorized as general Practitioner referral, emergency department, waiting list, booked or other. Data from the two sources were then merged based on a unique identifier using Microsoft excel.

To mitigate against the possible impact of knowledge of the project aims on study outcome, involved staff were not informed about the study aims and outcome. Phlebotomists were informed that an earlier phlebotomy ward round was to avoid clashes with clinical ward rounds and laboratory staff the need to process earlier sample delivery. Clinical staff were aware of introduction of the early phlebotomy round but were unaware (with the exception of coauthor SE) of the study objectives regarding impact on discharge time and rates. Data were collected retrospectively.

The main outcomes were the time between ward availability of pathology results and ward discharge time (shortened to "result availability to discharge time", or RADT) measured in days and hours, respectively. We also measured turnaround time (TAT) (specimen receipt to result availability) before and after the intervention.

In addition, we conducted a short patient survey on the two wards for the second cohort only. This was performed at the same time as the phlebotomy ward round and aimed to assess the impact on patients of the earlier phlebotomy ward round. The survey asked whether the patient was woken up for blood collection, whether they would be prepared to have an earlier blood collection (before 7:00 am) if it meant the possibility of early discharge, whether the blood collection interrupted their daily routine and how they would rate the blood collection experience out of 10 . The full survey is available on request from the authors.

\section{Ethical approval}

Not required as quality improvement project. Permission for data to be published has been granted by The Royal Wolverhampton NHS Trust Caldicott Guardian.

\section{Statistical analysis}

Prior to data analyses, we removed observations that were greater than three standard deviations away from the mean as these were considered outliers. These were all for the length of stay variable $(n=16)$, where patients stayed in hospital for over 45 days. We also removed observations where 
RADT was a negative value (i.e. the patient was discharged before the last blood sample was made available) $(n=7)$. There were no significant age or cohort year differences between outliers and nonoutliers, although outliers were slightly more likely to be female than male $(72.7 \%$ vs. $27.3 \%, p=$ 0.03). Removing observations left 655 patients for analysis.

We described patient characteristics descriptively and compared differences between the 2014 and 2015 cohort using $\chi 2$-analyses for categorical data (i.e. gender and admission type) and independent sample t-tests for normally distributed continuous data (i.e. age). This was to check the two cohorts were evenly balanced in terms of patient characteristics.

We then described differences between the two time periods in terms of the time at which samples were received into the laboratory and the time at which samples were made available to the wards. We also checked for differences in the time of discharge irrespective of the length of patient stay.

Finally, we tested for crude differences in RADT between the two samples using Wilcoxon signed rank tests (when RADT was measured in hours, i.e. a continuous variable) and $\chi 2$-tests (when RADT was measured in days, i.e. a categorical variable). We constructed regression models with RADT as the dependent variable and age, gender, admission type and length of admission as independent variables. The regression model was binary and logistic when RADT was measured in days, and linear when RADT was measured in hours. Regression models were run both unadjusted and adjusted for comparative purposes. In a sensitivity analysis, we reincluded outliers in the sample to test for the introduction of selection bias. We took an $\alpha$ value of 0.05 and reported $\beta$ coefficients with $95 \%$ confidence intervals $(95 \% \mathrm{Cls})$. All statistical analyses were conducted in Stata 15.0 (StataCorp).

\section{Results}

The total number of patients was 655: 345 in the 2014 cohort and 310 in the 2015 cohort. Patient characteristics in the two patient cohorts are shown in Table 1. The 2015 patient cohort compared to the 2014 patient cohort, was younger $(p<0.001)$ but had a similar gender mix. There were fewer general practitioner referrals and emergency department admissions but more waiting list admissions in the 2015 sample compared to the 2014 sample.

Following the intervention, samples were received into the laboratory earlier (Figure 1A), and laboratory results were available earlier (Figure 1B). Time of discharge, however, was unchanged (Figure 1C). Median (quartiles) specimen TATs were in 2014 (45 [39-54] min) and 2015 (40 [36-50] $\min )$. 
Table 1: Patient characteristics between the two samples.

Characteristic 2014 Sample $(n \square 345) 2015$ Sample p-Value $(n \square 310)$

\begin{tabular}{lrc}
\hline Age, years (mean, SD) & $68.2(18.0)$ & $63.0(16.1) \mathrm{p}<0.001^{\mathrm{a}}$ \\
Gender (\% male) & 50.8 & $49.8 \mathrm{p}=0.814^{\mathrm{b}}$ \\
$\begin{array}{l}\text { Admission method } \\
\quad \text { General practitioner }\end{array}$ & 20.0 & $14.2 \mathrm{p}=0.019^{\mathrm{b}}$ \\
$\quad$ referral, \% & & \\
$\quad$ Emergency department, \% & 40.9 & 34.8 \\
$\quad$ Waiting list, \% & 30.1 & 40.3 \\
Booked, \% & 2.9 & 5.2 \\
Other, \% & 6.1 & 5.5 \\
\hline
\end{tabular}

andependent samples t-test; ${ }^{\mathrm{b}} \chi^{2}$-test.

Dichotomizing RADT into same day discharge versus non-same day discharge, more patients were discharged on the same day as test result availability in 2015 (64\%) than in 2014 (52\%) as shown in Figure 2. This pattern retained statistical significance on an unadjusted logistic regression model (OR $=1.66,95 \% \mathrm{Cl}=1.21-2.27, \mathrm{p}=0.002)$ and after adjustment for age, gender, admission type and length of admission ( $A O R=1.68,95 \% \mathrm{Cl}=1.20-2.34, p=0.002)$. RADT as a continuous variable, in 2015 (median = 9.62, IQR = 7.23-30.40 h) and 2014 (median = 19.83, IQR = 5.82-30.28 h), were similar (Mann-Whitney test: $z=-0.848, p=0.396$ ). The lack of statistical significance was present in an unadjusted linear regression model ( $\beta$ coefficient $[B]=-0.06,95 \% \mathrm{Cl}=-0.21,0.09, p=0.406$ ) and after adjustment for age, gender, admission type and length of admission $(B=-0.08,95 \% \mathrm{Cl}=-0.23$, $0.08, p=0.340$ ).

All regression model outputs remained unchanged after including the 23 outlier patients that were originally removed prior to analysis.

\section{Patient survey}

Following the intervention, 20 patients (nine males, 11 females) were surveyed. Nineteen patients reported already being awake at the time of blood collection, all patients were prepared to have an earlier blood test if it meant the possibility of early discharge and no patients felt the earlier blood test interfered with their daily routine. Patients scored a mean 8.7 out of 10 for the experience of their earlier blood test.

Anecdotal conversations with ward staff, phlebotomists, runners and laboratory personnel indicated that for those involved they preferred the early start and respective early finish. 

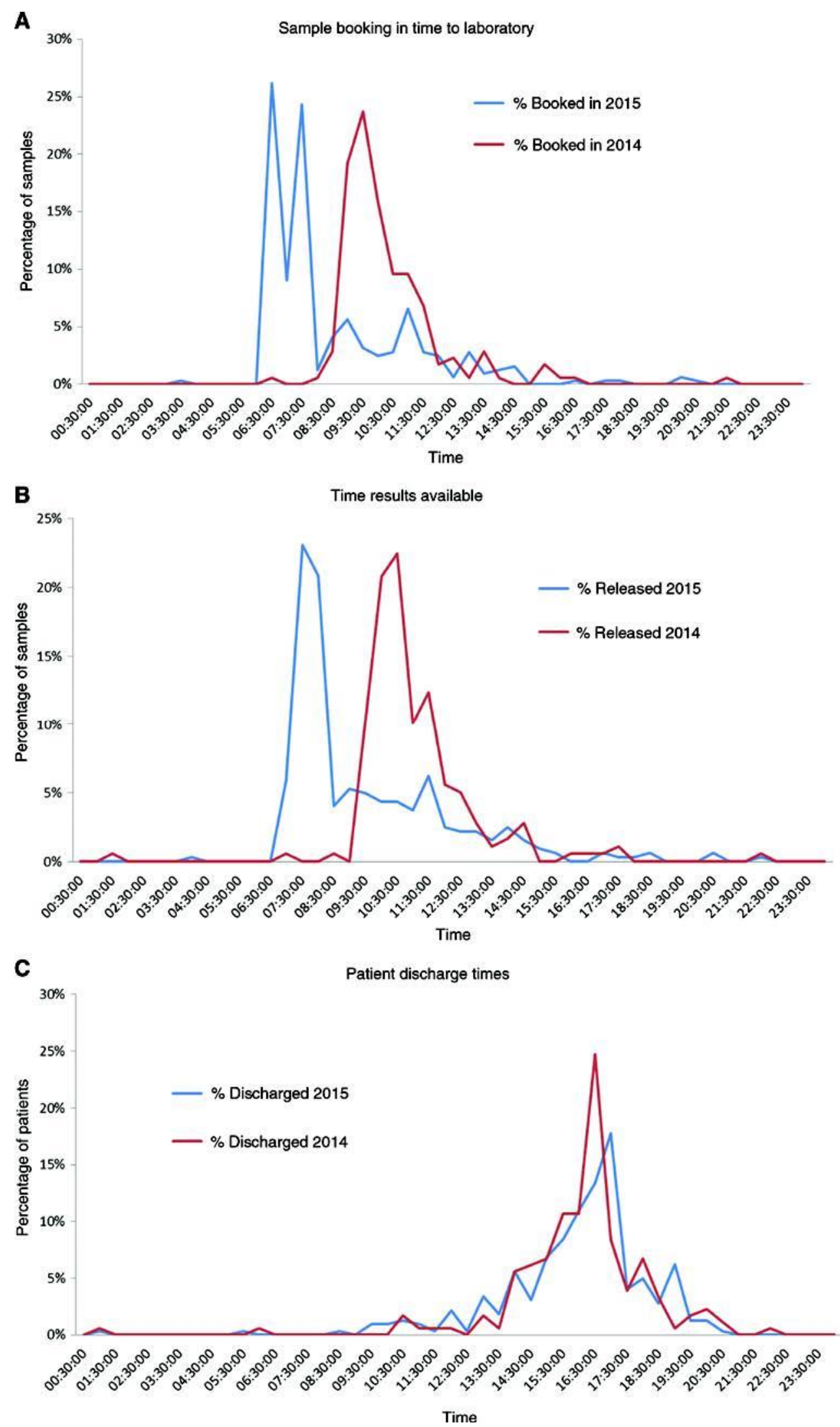

Figure 1: Graphs showing sample processing in 2015 vs. 2014. Graph (A) shows the booking in time of samples. Graph (B) shows the time results were available to the clinicians. Graph (C) shows the time patients are discharged. 


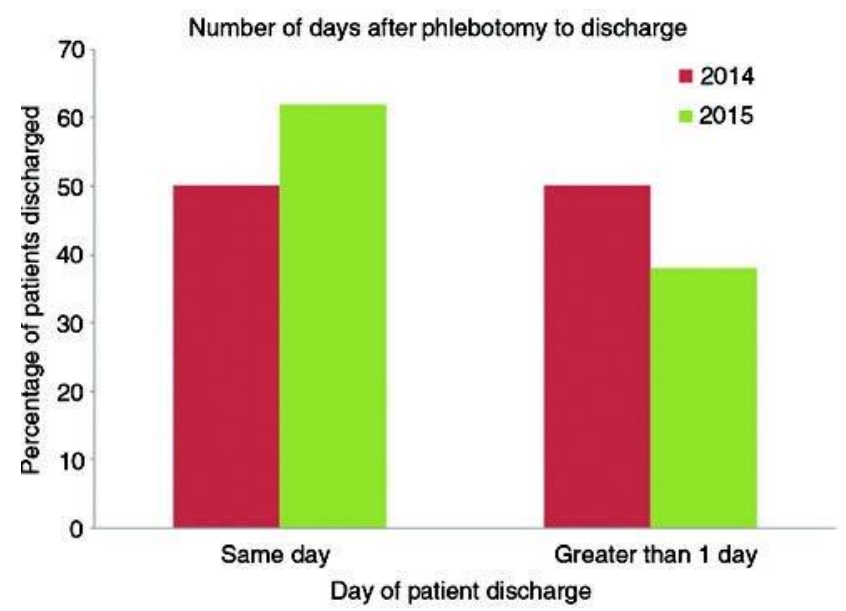

Figure 2: The effect of earlier phlebotomy on day of discharge.

\section{Discussion}

Early phlebotomy resulted in earlier receipt of samples in the laboratory (Figure $1 \mathrm{~A}$ ) facilitating earlier sample processing and analysis with results being available $2 \mathrm{~h}$ earlier in 2015 compared to 2014. Over $95 \%$ of results were available before 08.30 in 2015 as compared to less than $1 \%$ in 2014 (Figure 1B). This greater than 2-h improvement in results availability despite earlier phlebotomy round starting only $1 \mathrm{~h}$ earlier was due to samples being delivered earlier to the laboratory after collection because TAT (specimen receipt to result availability; TAT) was unaffected by the intervention. Same day discharge rates significantly increased after earlier result availability (Figure 2 ), but time of discharge was unchanged (Figure 1C). These effects were unrelated to seasonal variability because the two study periods, 1 year apart, were identical. A higher proportion of patients were planned admissions in 2015 compared to 2014 (Table 1), offering a potential explanation for the increased discharge rates in 2015 because elective surgery patients would generally be fitter and have fewer complications. The number of same day discharges, however, remained significantly increased following the intervention after adjustment for age, gender, and type and length of admission. Time to discharge was shorter in 2015 (median $=9.62$ vs. 19.83, Figure 1C) than 2014 , but this was not statistically significant even after adjustment for age, gender, admission type and length of admission.

TAT time, as previously specified, was unaffected by earlier phlebotomy, indicating that TAT following routine phlebotomy was already optimal in our laboratory. The increase in same day ward discharges, in our hospital, could therefore only be achieved by early phlebotomy and consequent earlier test result availability.

This study supports the notion that blood test result availability may have an impact on ward discharges. It is consistent with studies reporting that diagnostic service delays contribute to delayed discharge $[4,6,11]$ but not with others reporting no impact $[7,8]$. In this study, we evaluated the impact of just blood science results on discharge, whereas others have studied the impact of 
diagnostic services, largely radiology and all pathology disciplines (blood sciences, microbiology and histopathology). The differences in these studies may, therefore, be lack of clarity regarding the contribution of pathology (and its individual disciplines) to diagnostic services, particularly because the contribution of diagnostic services delaying discharge appears to be outside of pathology [11]. These differences may also be due to variations between UK $[7,8]$ and non-UK $[4,6,10]$ healthcare provision. Future such studies should clearly define what aspects of diagnostic medicine or pathology are being evaluated.

The increased same day discharge rate but unchanged time of discharge suggests that discharge time may be relatively fixed for patient and provider convenience and therefore may not be a useful key performance indicator. Other factors, beyond the scope of this service evaluation, may also be relevant in determining discharge time. These include waiting for discharge medication from pharmacy, physiotherapy assessment, transport or family to collect the patient as well as social care availability and time to complete the required documentation or any combination of these factors. Further studies on discharge time should focus on these factors to identify areas for improvement to enhance cost-effective healthcare delivery.

There are limitations to this study. We did not publicize the potential for earlier discharge with earlier availability of blood results because, as previously alluded, we wished to avoid outcome bias from prior knowledge of study aims. Although clinical staff were aware of early phlebotomy and test result availability, at the time of the study we did not have the facility to audit the time when results were accessed by clinicians. It is, therefore, possible that although test results were available earlier, they were accessed at same time before and after the intervention.

Early phlebotomy was popular with patients, ward staff, phlebotomists and laboratory personnel. The patient survey indicated patients were nearly all awake and overwhelmingly content with an earlier blood draw. Patients scored their phlebotomy experiences very highly and were very grateful for the care they were receiving. Ward staff preferred early phlebotomy as this no longer disrupted breakfast and reduced clashes between different staff groups performing their respective duties. Laboratory staff volunteered for the earlier start as they preferred to finish earlier as this was conducive to their lifestyle.

There were no cost implications to either the ward or laboratory for an intervention of this scale. Work pattern changes were achieved by a redistribution existing staff by altering shift patterns. Routinely monitored laboratory key performance indicators, including sample rejection rates and TAT, were similar in the two study periods indicating that quality of the service was maintained.

In summary, we have provided an early phlebotomy round with earlier availability of blood results prior to ward rounds, which significantly increased ward discharges at no extra cost to the laboratory. It was popular with patients, ward staff, phlebotomists and laboratory personnel and therefore considered a service quality improvement, and its implementation has remained in place after the study.

Acknowledgments: Helen Latunji Royal Wolverhampton NHS Trust Information department Royal Wolverhampton NHS Trust Phlebotomy Team.

Author contributions: $\mathrm{MC}, \mathrm{GD}, \mathrm{RG}, \mathrm{CF}$ and SE designed the study. MC coordinated the study. Data were collected by ER, JT and MC. GD conceived the study. Data analysis was performed by MC and MJ. The manuscript was drafted by MC, RG, MJ and CF. All authors reviewed and added to the final 
draft. All the authors have accepted responsibility for the entire content of this submitted manuscript and approved submission.

Guarantor: RG.

Research funding: This quality improvement project received no grant from any funding agency in the public, commercial or not-for-profit sectors. The authors received no financial support for the authorship and/or publication of this article. The Public Health Policy Evaluation Unit at Imperial College London is supported by funding from the NIHR School of Public Health Research.

Employment or leadership: None declared.

Honorarium: None declared.

Competing interests: The funding organization(s) played no role in the study design; in the collection, analysis, and interpretation of data; in the writing of the report; or in the decision to submit the report for publication.

\section{References}

1. Bryan K. Policies for reducing delayed discharge from hospital. Br Med Bull 2010;95:33-46.

2. National Audit Office. Inpatient admissions and Bed Management in NHS Acute Hospitals. London: Stationary Office, 2000.

3. Khanna S, Sier D, Boyle J, Zeitz K. Discharge timeliness and its impact on hospital crowding and emergency department flow performance. Emerg Med Australas 2016;28:164-70.

4. Selker HP, Beshansky JR, Pauker SG, Kassirer JP. The epidemiology of delays in a teaching hospital. The development and use of a tool that detects unnecessary hospital days. Med Care 1989;27:11229.

5. Carey MR, Sheth H, Braithwaite RS. A prospective study of reasons for prolonged hospitalizations on a general medicine teaching service. Gen Intern Med 2005;20:108-15.

6. Silva SA, Valácio RA, Botelho FC, Amaral CF. Reasons for discharge delays in teaching hospitals. Rev Saude Publica 2014;48:314-21.

7. Hendy P, Patel JH, Kordbacheh T, Laskar N, Harbord M. In-depth analysis of delays to patient discharge: a metropolitan teaching hospital experience. Clin Med 2012;12:320-3.

8. Majeed MU, Williams DT, Pollock R, Amir F, Liam M, Foong KS, et al. Delay in discharge and its impact on unnecessary hospital bed occupancy. BMC Health Serv Res 2012;12:410.

9. First steps in improving phlebotomy: the challenge to improve quality, productivity and patient experience. http://www.apho.org.uk/resource/view.aspx?RID=171742. Accessed: 6 Apr 2017.

10. Ou L, Young L, Chen J, Santiano N, Baramy LS, Hillman K. Discharge delay in acute care reasons and determinants of delay in general ward patients. Aust Health Rev 2009;33:513-21.

11. Conway R, Murray FE. Assessment of service delays and impact on bed utilisation in a major teaching hospital. Ir J Med Sci 2011;180:533-5. 